\title{
artigo
}

Ribeiro, S.H.M.G.; Bueno da Silva, M.; Cerqueira, M.D.R.A.; Castro, R.C.; Quitete, J.B.; Knupp, V.M.A.O.;

Perfil epidemiológico de mulheres que sofreram violência obstétrica: estudo transversal

\section{Perfil epidemiológico de mulheres que sofreram violência obstétrica: estudo transversal}

\author{
Epidemiological profile of women who suffered obstetric violence: a cross-sectional study. \\ Perfil epidemiológico de las mujeres que sufrieron violencia obstétrica: un estudio transversal
}

\begin{abstract}
RESUMO
Objetivo: analisar o perfil epidemiológico da mulher que foi submetida a violência obstétrica. Método: Trata-se de um estudo transversal tendo como participantes mulheres vítimas de violência obstétrica em uma região da Baixada Litorânea do Estado do Rio de Janeiro, no ano de 2018. Resultados: Foram entrevistadas 33 mulheres, das quais a idade mínima foi de 18 anos, a máxima 39 anos e a média de 27 anos. Os dados revelam maior proporção entre as mulheres com ensino médio completo, via de parto cesárea, com apenas um parto anterior, que não tiveram direito a um acompanhante, e que foram assistidas pelo Sistema Único de Saúde. Conclusão: 0 presente estudo corrobora com achados já publicados e evidencia a necessidade de um contínuo exercício de qualificação por parte dos profissionais de saúde que assistem mulheres durante a gravidez, parto e puerpério, com subsídios das evidências cientificas.
\end{abstract}

DESCRITORES: Saúde da Mulher; Violência contra a Mulher; Epidemiologia; Parto; Direitos Reprodutivos.

\section{ABSTRACT}

Objective: to analyze the epidemiological profile of women who are victims of obstetric violence. Methods: This is a cross-sectional study with the participants of women victims of obstetric violence in a region of the coastal lowland of the state of Rio de Janeiro, in 2018. Results: Thirty-three women were interviewed, of whom the minimum age was 18 years, the maximum was 39 years and the average was 27 years. The data reveal a higher proportion among women with complete high school, by cesarean delivery, with only one previous delivery, who were not entitled to a companion, and who were assisted by the Unified Health System. Conclusion: The present study corroborates with already published findings and evidences the need for a continuous exercise of qualification by health professionals who assist women during pregnancy, childbirth and puerperium, with subsidies from scientific evidence.

DESCRIPTORS: Women's Health; Violence Against Women; Epidemiology; Parturition; Reproductive Rights.

\section{RESUMEN}

Objetivo: analizar el perfil epidemiológico de las mujeres que han sido sometidas a violencia obstétrica. Método: Este es un estudio transversal con las participantes de mujeres víctimas de violencia obstétrica en una región de las Tierras Bajas Costeras del Estado de Río de Janeiro, en 2018. Resultados: Se entrevistó a treinta y tres mujeres, de las cuales la edad mínima era de 18 años, el máximo era de 39 años y el promedio era de 27 años. Los datos revelan una mayor proporción entre las mujeres con secundaria completa, por parto por cesárea, con sólo un parto previo, que no tenían derecho a un compañero, y que fueron asistidas por el Sistema Unificado de Salud. Conclusión: El presente estudio corrobora con hallazgos ya publicados y evidencia la necesidad de un ejercicio continuo de cualificación por parte de profesionales de la salud que ayudan a las mujeres durante el embarazo, el parto y el puerperium, con subsidios de evidencia científica.

DESCRIPTORES: Salud de la Mujer; Violencia contra la Mujer; Epidemiología; Parto; Derechos Reproductivos.

RECEBIDO EM: 15/03/2021 APROVADO EM: 31/03/2021

\section{Sandro Henrique Miranda Gonçalves Ribeiro}

Residente em Enfermagem Obstétrica, pela Universidade Federal Fluminense (UFF) - Niterói (RJ), Brasil.

ORCID: 0000-0001-8720-2198

\section{Miriellen Bueno da Silva}

Residente em Enfermagem em Saúde Coletiva, pela Universidade Federal Fluminense (UFF) - Niterói (RJ), Brasil.

ORCID: 0000-0001-5353-2412 


\section{Mara Dayanne Ramos Alves de Cerqueira}

Residente em Enfermagem Obstétrica, pelo Centro Universitário Arthur Sá Earp Neto UNIFASE/FMP, Petrópolis (RJ), Brasil. ORCID: 0000-0003-1903-8298

\section{Rosana de Carvalho Castro}

Universidade Federal Fluminense (UFF) - Rio das Ostras (RJ), Brasil.

ORCID: 0000-000379-9244

\section{Jane Baptista Quitete}

Universidade Federal Fluminense (UFF) - Rio das Ostras (RJ), Brasil.

ORCID: 0000-0003-0330-458X

\section{Virginia Maria de Azevedo Oliveira Knupp}

Universidade Federal Fluminense (UFF) - Rio das Ostras (RJ), Brasil.

ORCID: 0000-0001-5512-2863

\section{INTRODUÇÃO}

A partir do século XX o parto passa a ser predominantemente hospitalar e privativo da medicina. $\mathrm{O}$ ato de parir passou a ser visto como patológico e potencialmente de risco perdendo sua essência natural, dessa forma a mulher torna-se esquecida e tomada como objeto e não como sujeito, na qual ela perde sua autonomia nesta relação. Tal fato faz com que o seu corpo e sua sexualidade sejam controlados pela medicina ${ }^{(1)}$.

De acordo com pesquisa realizada pela Fundação Perseu Abramo, uma em cada quatro brasileiras é vítima de violência obstétrica ${ }^{(2)}$. A realidade obstétrica brasileira caracteriza-se por um atendimento com grande número de intervenções cirúrgicas, que por muitas vezes torna-se humilhante e até a negação do direito ao acompanhante. Sendo assim um desrespeito às mulheres, além de ser contra os direitos humanos ${ }^{(3)}$. A presença do acompanhante é um direito garantido pela Lei $\mathrm{n}^{\circ} 11.108 / 2005^{(4)}$, observa-se que por diversas vezes esse direito não é informado à mulher e, consequentemente não é garantido durante o trabalho de parto, parto e pós-parto em inúmeras instituições hospitalares públicas e privadas.

A violência pode trazer consequências adversas na saúde física, mental, sexual e reprodutiva destas mulheres ${ }^{(5)}$. A expressão "violência obstétrica" é parte de reivindicações do movimento feminista local e de processo institucional da violência con- tra a mulher como um problema social, político e público. Nesse sentido, pode-se entender que o problema da violência obstétrica é resultado de uma precariedade no sistema de saúde, que faz restrição considerável aos serviços oferecidos além de promover práticas desrespeitosas e desumanas na assistência a mulher no processo de parto e nascimento ${ }^{(6)}$.

Diante disso, o presente estudo teve como objetivo analisar o perfil epidemiológico da mulher que foi submetida a violência obstétrica.

\section{MÉTODO}

Trata-se de um estudo transversal realizado com mulheres que passaram pela experiência da violência obstétrica nos seus partos em um município da Baixada
Litorânea do Estado do Rio de Janeiro no ano de 2018. A coleta de dados foi realizada por meio de um questionário com perguntas fechadas, aplicado às participantes do estudo.

A amostra do estudo foi selecionada em um grupo de gestante denominado Gestante de Vida: espaço de empoderamento feminino, projeto coordenado por docentes e discentes do Curso de Enfermagem/Universidade Federal Fluminense/Campus de Rio das Ostras (Figura 1).

O estudo foi aprovado pelo comitê de Ética (CEP) do Hospital Universitário Antônio Pedro (HUAP), e faz parte da pesquisa intitulada "Violência Institucional Obstétrica à Parturiente nos serviços Públicos de Saúde: A vivência das Mulheres", CAAE $n^{\circ} 1.109 .636$ de acordo com a Resolução no 466/2012 do Conselho Na-

\section{Figura 1 - Fluxograma da amostra do estudo.}

Parturientes atendidas em

2018. 960 mulheres

\section{Vítimas de violência Obstétrica: 672}

\section{Excluídas 639}

\section{Amostra 33}


Figura 2 - Boxplot da distribuição da idade das mulheres entrevistadas em Rio das Ostras em 2019.

\section{Boxplot de Idade}

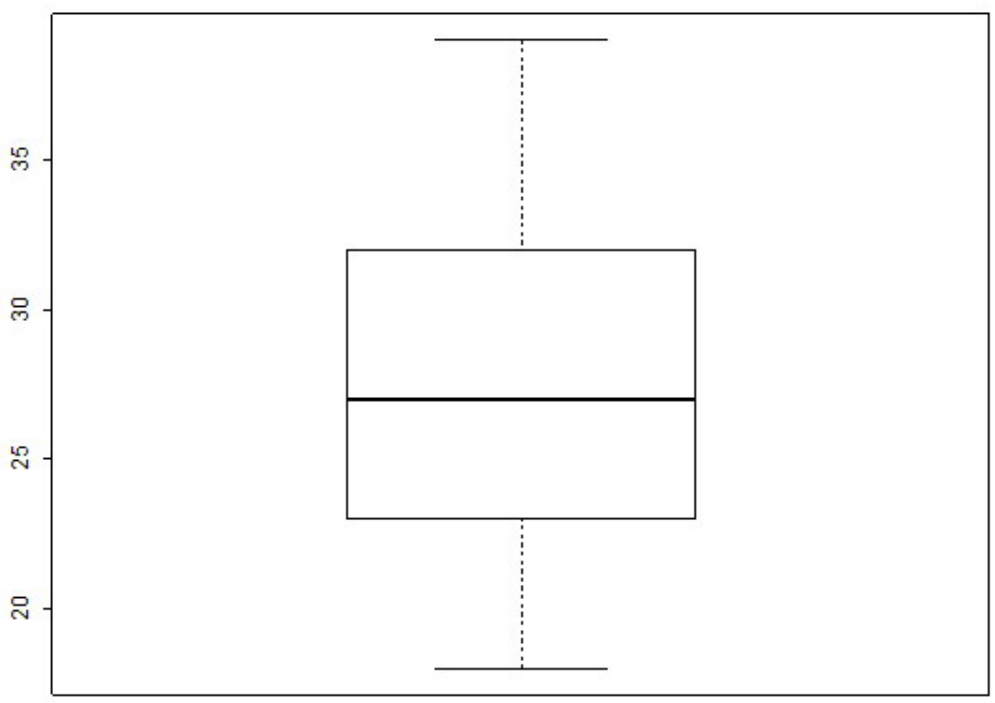

Fonte: elaborado pelos autores.

Tabela 1 - Distribuição das variáveis sociais e relacionadas a gravidez das mulheres de Rio das Ostras em 2019.

\section{VARIÁVEIS}

$\begin{array}{ccc}\text { Idade } & \mathrm{N}=33 & \% \\ \text { Até } 19 \text { anos } & 2 & 6,1 \\ \text { 20 a } 25 \text { anos } & 11 & 33,3 \\ \text { 26 a } 30 \text { anos } & 9 & 27,3 \\ \text { 31 a } 35 \text { anos } & 8 & 24,2 \\ \text { 36 a } 39 \text { anos } & 3 & 9,1 \\ \text { Escolaridade } & \mathrm{N}=33 & \% \\ \text { Ensino fundamental incompleto } & 5 & 15,2 \\ \text { Ensino fundamental completo } & 2 & 6,1 \\ \text { Ensino médio incompleto } & 10 & 30,3 \\ \text { Ensino médio completo } & 13 & 39,4 \\ \text { Ensino superior incompleto } & 1 & 3,0 \\ \text { Ensino superior completo } & 2 & 6,1 \\ \text { Via de parto } & \mathrm{N}=33 & \% \\ \text { Vaginal } & 13 & 39,4 \\ \text { Cesárea } & 14 & 42,4 \\ \text { Vaginal e Cesárea } & 5 & 15,2 \\ \text { Sem informação } & 1 & 3,0 \\ \text { Gesta } & \mathrm{N}=33 & \%\end{array}$

cional de Saúde (CNS), que regulamenta a pesquisa envolvendo seres humanos ${ }^{(7)}$, foi esclarecido a todas as participantes, sobre possíveis desconfortos e riscos decorrentes da participação, bem como, sobre os benefícios esperados. Garantido sigilo e privacidade das participantes.

Foram considerados como critérios de inclusão: ser maior de 18 anos, ter o desejo de participar da pesquisa, ter tido pelo menos um parto no município de Rio das Ostras. Foram excluídas da pesquisa as participantes que tiveram complicações obstétricas durante a gestação, trabalho de parto e/ou parto, bem como as mulheres que tiveram partos prematuros, natimorto e/ou neomorto. Os dados coletados foram digitados em planilha eletrônica e processados no Programa R, que é gratuito e disponível para download em http://cran.r-project. org/mirrors.html. Foi calculada a proporção e as medidas de tendência central.

\section{RESULTADOS}

Foram entrevistadas 33 mulheres, das quais a idade mínima foi de 18 anos, a máxima 39 anos e a média de 27 anos (Figura 2). O estrato etário com menor proporção foi observado no grupo com até de 19 anos $(6,1 \%)$ e o maior de 20 a 25 anos (33,3\%). Em relação à escolaridade, verificou-se a maior proporção entre as mulheres com ensino médio completo (39,4\%) e a menor entre aquelas com ensino fundamental completo $(6,1 \%)$ e ensino superior completo $(6,1 \%)$. (Tabela 1$)$.

$\mathrm{Na}$ análise da via de parto, a diferença foi pequena entre vaginal e cesárea, mas com maior proporção para cesárea $(42,4 \%)$. Em relação ao número de gestações (GESTA), a maior proporção foi observada entre aquelas com uma gestação $(30,3 \%)$ e a menor entre as com cinco gestações $(6,1 \%)$. Em relação ao número de partos (PARA), verificou-se a maior proporção entre aquelas com um parto $(39,4 \%)$ e a menor entre quatro e cinco, com $3 \%$ em cada estrato (Tabela 1).

A análise das variáveis relacionadas ao local do parto e a ocorrência de violência obstétrica, verificou-se que $84,8 \%$ dos par- 
Tabela 2 - Distribuição das variáveis relacionadas à violência obstétrica das mulheres de Rio das Ostras em 2019.

\section{VARIÁVEIS}

Natureza jurídica do local do parto

Público

Privado

Sabe o que é violência obstétrica

$\operatorname{Sim}$

Não

Já sofreu alguma violência obstétrica

Sim

Não

Não sabe

Quando sofreu

Pré-parto

Parto

Pré-parto e parto

Puerpério

Não se aplica

Foi informada dos direitos durante todo o trabalho de parto

Sim

Não

Não sabe informar

Permitido a presença de um acompanhante de sua escolha

Sim

Não

Não sabe

Conhece a Lei do Acompanhante n 11.108

Sim

Não

Fonte: elaborado pelos autores.

Tabela 3 - Distribuição das variáveis relacionadas à assistência recebida pelas entrevistadas em Rio das Ostras em 2019.

\section{VARIÁVEIS}

Classificação da assistência recebida

$\begin{array}{cc}\mathrm{N}=33 & \text { \% } \\ 12 & 36,4 \\ 8 & 24,2 \\ 1 & 3,0\end{array}$

tos aconteceram em estabelecimento de saúde público. Em relação ao recebimento de informação durante todo o trabalho de parto, a maioria afirmou que $\operatorname{sim}(63,6 \%)$. Quanto à presença de acompanhante durante todo o trabalho de parto, verificou-se que a maioria respondeu não $(42,4 \%)$ (Tabela 2).

Em relação à assistência recebida pelos profissionais de maneira geral no momento do trabalho de parto, a maioria respondeu como excelente $(36,4 \%)$ e boa (24,2\%). Quando questionadas se pudesse escolher a instituição que quer ter outro parto e se voltaria a instituição do parto anterior, $48,5 \%$ disse que sim, mas merece destaque o estrato "não gostaria de ter outro parto" $(12,1 \%)$ e "não haveria esta possibilidade $(6,1 \%)$. A maioria relatou que não se sentiu traumatizada $(78,8 \%)$ e que não afetou o seu relacionamento com sua família e bebê (90,9\%) (Tabela 3).

\section{DISCUSSÃO}

O perfil epidemiológico das mulheres vítimas de violência obstétrica tem relação com questões socioeconômicas, físicas e culturais. Os resultados apontam que, entre as mulheres entrevistadas a maioria tem idade entre 20 a 25 anos e baixa escolaridade. Entre as mulheres entrevistadas, é possível notar que a percepção em saúde a respeito do tema abordado pode ser comprometida, tendo em vista as questões que abrangem o nível de escolaridade ou idade das participantes, acredita-se que a mulher não perceba a sua real situação frente a violência obstétrica.

É importante entender, que a permanência de atos violentos no tocante a assistência obstétrica é caracterizada, pelo desconhecimento da mulher em relação aos seus direitos sexuais e reprodutivos ${ }^{(8)}$. No Brasil, grande parte dos partos hospitalares, são realizados por médicos obstetras, profissionais com formação tecnicista e intervencionista, que estão associado ao aumento da incidência de cesarianas ${ }^{(9)}(10)$.

A questão da grande prevalência de cesarianas no município de Rio das 


\begin{tabular}{|c|c|c|}
\hline Regular & 6 & 18,2 \\
\hline Regular e ruim & 1 & 3,0 \\
\hline Ruim & 5 & 15,2 \\
\hline Voltaria a instituição do parto anterior & $\mathrm{N}=33$ & $\%$ \\
\hline $\operatorname{Sim}$ & 16 & 48,5 \\
\hline Não & 8 & 24,2 \\
\hline Não gostaria de ter outro parto & 4 & 12,1 \\
\hline Não haveria esta possibilidade & 2 & 6,1 \\
\hline Talvez & 3 & 9,1 \\
\hline Você se sentiu traumatizada & $\mathrm{N}=33$ & $\%$ \\
\hline $\operatorname{Sim}$ & 6 & 18,2 \\
\hline Não & 26 & 78,8 \\
\hline Prefiro não opinar & 1 & 3,0 \\
\hline Afetou o seu relacionamento com sua família e bebê & $\mathrm{N}=33$ & $\%$ \\
\hline $\operatorname{Sim}$ & 2 & 6,1 \\
\hline Não & 30 & 90,9 \\
\hline Somente com alguém da família & 1 & 3,0 \\
\hline
\end{tabular}

Ostras é um dado alarmante que pode caracterizar uma assistência voltada ao modelo hospitalocêntrico no qual o médico é o único profissional responsável pelo trabalho de parto e parto ${ }^{(11)}$. Tal fato contraria o que é preconizado pela OMS desde 1985, que é considerado como a taxa ideal para procedimento de cesárea de 10 a $15 \%$ entre os partos realizados, bem como recomenda a inserção de enfermeiras obstétricas na assistência as parturientes de risco habitual ${ }^{(12)}$.

Quando não se tem uma indicação obstétrica, a cesariana pode ocasionar riscos evitáveis à saúde da mulher e do bebê, que pode aumentar em até 120 vezes a probabilidade de afecções respiratórias para o recém-nascido e pode triplicar o risco de óbito para a mãe. Os principais riscos são a infecção e complicações cirúrgicas, em instituições com uma infraestrutura ineficaz ou incapaz de fornecer uma cirurgia segura ${ }^{(9)}$.

É perceptível a violência física e a violação do direito à informação e autonomia, quando são realizadas intervenções e práticas que podem ser consideradas prejudiciais cientificamente, sem a autorização da parturiente ou quando são autorizadas mediante as informações distorcidas e in-
Avançar na discussão

sobre o perfil de mulheres que podem

ser vítimas de

violência obstétrica é

de suma importância,

para que através

de estudos como

este tenhamos uma

assistência mais

humanizada no

momento do parto. completas, por exemplo: mentir sobre a dilatação da paciente, a vitalidade fetal, e motivos para indicação de cesariana improcedentes, entre outros ${ }^{(13)}$. Através destes fatos apresentados pelos estudos citados, é possível entender que uma cirurgia de cesariana, sem a indicação adequada para a tal, pode ser caracterizada como uma violência obstétrica ${ }^{(14)}$.

Os resultados evidenciam que a grande maioria dos partos realizados no município de Rio das Ostras foi em instituição pública. Do total de mulheres entrevistadas a maioria afirma conhecer o que é violência obstétrica. No entanto, percebemos uma contradição no decorrer das entrevistas, pois, quando perguntadas se tiveram informações a respeito dos seus direitos durante todo o trabalho de parto e se foram permitidos a elas a presença de um acompanhante de sua escolha. Ao serem questionadas sobre o conhecimento acerca da Lei do Acompanhante foi verificado que mais de $60 \%$ não tem o conhecimento sobre esta legislação.

No momento do parto, a mulher precisa de atenção e explicações sobre os procedimentos e intervenções que serão realizados, respeito e empatia, e mais importante, a possibilidade de ter uma participação efetiva nesta fase de sua vida ${ }^{(15)}$. O conhecimento das mulheres acerca da violência obstétrica, não é eficaz, pois a grande maioria não tem conhecimento de fatores que podem caracterizar uma violência durante o trabalho de parto $\mathrm{e}$ o parto, que pode ser ilustrado ao afirmarem que não receberam informações suficientes sobre seus direitos durante a internação hospitalar ${ }^{(16)}$. Deste modo, tornam-se vulneráveis.

Avançar na discussão sobre o perfil de mulheres que podem ser vítimas de violência obstétrica é de suma importância, para que através de estudos como este tenhamos uma assistência mais humanizada no momento do parto. A limitação deste estudo ocorreu na dificuldade de acesso às mulheres entrevistadas, bem como na interpretação dos dados obtidos pela coleta de dados. 


\section{CONCLUSÃO}

O presente estudo corrobora com achados já publicados e evidencia a necessidade de um contínuo exercício de qualificação por parte dos profissionais de saúde que assistem mulheres durante a gravidez, parto e puerpério, com subsídios das evidências cientificas.

Enfatizamos a incorporação de mudanças na formação acadêmica de maneira a incorporar as evidências científicas nos currículos de medicina e enfermagem, bem como, uma mudança urgente nas normas e rotinas hospitalares, abolindo procedimentos intervencionistas e colocando a mulher na posição de protagonista do seu processo de parir, com empoderamento e participação na tomada das decisões. -

\section{REFERÊNCIAS}

1. Barboza, LP, Mota, A. VIOLÊNCIA OBSTÉTRICA: VIVÊNCIAS DE SOFRIMENTO ENTRE GESTANTES DO BRASIL. Revista Psicologia, Diversidade e Saúde, [S.I.], v. 5, n. 1, maio 2016. ISSN 23173394. [Acesso em: 23 de abril de 2020]. Disponivel em: https:// www5.bahiana.edu.br/index.php/psicologia/article/view/847 .

2. BRASIL. Ministério da Saúde. Secretaria de Ciência, Tecnologia e Insumos Estratégicos. Departamento de Gestão e Incorporação de Tecnologias em Saúde. Diretrizes Nacionais de Assistência ao Parto Normal. (Versão Resumida). Brasília - DF, 2017. [Acesso em: 23 Mar. 2020]. Disponivel em: http://bvsms. saude.gov.br/bvs/publicacoes/diretrizes_nacionais_assistencia_parto_normal.pdf .

3. Zanardo GLP, Uribe MC, Nadal AHR, Habigzang LF. Violência Obstétrica no Brasil: Uma Revisão Narrativa. Psicologia \& Sociedade, 29: e155043. Pontifícia Universidade Católica do Rio Grande do Sul, Porto Alegre/RS, Brasil, 2017. [Acesso em: 25 de abril de 2020]. Disponivel em: http://www.scielo.br/pdf/psoc/ v29/1807-0310-psoc-29-e155043.pdf .

4. BRASIL. Lei $N^{\circ} 11.108$, de 7 de Abril de 2005. Altera a lei $n^{\circ}$ 8.080, de 19 de setembro de 1990, para garantir às parturientes o direito à presença de acompanhante durante o trabalho de parto, parto e pós-parto imediato, no âmbito do sistema único de saúde - sus. Diário Oficial [da] União de 08 de Abril de 2005, p.1. [Acesso em: 23 Mar. 2020]. Disponivel em: http://legislacao. planalto.gov.br/legisla/legislacao.nsf/Viw_Identificacao/lei\%20 11.108-2005?OpenDocument .

5. Pereira JSS, Silva JCO, Borges NA, Ribeiro MMG, Auarek LJ, Souza JHK . Obstetrical Violence: Offense for dignity. Brazilian Journal of Surgery and Clinical Research - BJSCR. [Internet]. JunAgo 2016. [Acesso em: 2020 jul 04]; 15 (1): 103-108. Disponível em: http://www.repositorio.ufop.br/handle/123456789/6646 .

6. Rodrigues $D$, Alves $V$, Vieira $R$, Leão $D$, Paula $E$, Pimentel $M$. Violência obstétrica no contexto do parto e nascimento. Revista de Enfermagem UFPE on line [Internet]. 01 de janeiro de 2018; [Citado em 04 de julho de 2020]; 12 (1): 236-246. Disponivel em: https://periodicos.ufpe.br/revistas/revistaenfermagem/article/ view/23523.

7. BRASIL. Ministério da Saúde. Conselho Nacional de Saúde. Publicada Resolução 466 do CNS que trata de pesquisas em seres humanos e atualiza a resolução 196. Brasília, 2013. [Acesso em: 23 Mar. 2020]. Disponivel em: http://conselho.saude.gov. br/ultimas_noticias/2013/06_jun_14_publicada_resolucao. html.

8. Jardim DMB, Modena CMA. Violência obstétrica no cotidiano assistencial e suas características. Rev. Latino-Am. Enfermagem, v. 26, Ribeirão Preto, 2018. [Acesso em: 2020 jun 29]
Epub 29 Nov 2018. Disponivel em: http://www.scielo.br/scielo. php?script=sci_arttext\&pid=S0104-11692018000100613\& $\mathrm{I}-$ $n g=p t \& n r m=i s o \&$ tlng=pt .

9. Souza AB, Silva LC, Alves RN, Alarcão ACJ. Fatores associados à ocorrência de violência obstétrica institucional: uma revisão integrativa de literatura. Revista de Ciências Médicas, Campinas, [Acesso em: 2020 jun 30] 25(3):115-128, set/dez, 2016. Disponivel em: https://seer.sis.puc-campinas.edu.br/seer/index. php/cienciasmedicas/article/view/3641/2486.

10. Leal MC, Gama SGN. NASCER NO BRASIL. Inquérito Nacional sobre parto e nascimento. [Acesso em: 2020 jan 26] Disponível em: http://www.ensp.fiocruz.br/portal-ensp/informe/site/arquivos/anexos/nascerweb.pdf .

11. Sales JL, Quitete JB, Knupp VMAO, Martins MAR. Assistência ao parto em um hospital da baixada litorânea do Rio de Janeiro: desafios para um parto respeitoso. Rev Fun Care Online. 2020 jan/dez; 12:107-113. DOI: http://dx.doi.org/10.9789/21755361.rpcfo.v12.7092.

12. WHO. Declaração da OMS sobre Taxas de Cesáreas. Organização Mundial de Saúde. 2015. [Acesso em: 2020 jun 29] Disponivel em: https://apps.who.int/iris/bitstream/handle/10665/161442/WHO_RHR_15.02_por.pdf;jsessionid =91F03E2F4FA98D2D495FFEE3F289CCA5?sequence $=3$.

13. Brandt GP, Souza SJP, Migoto MT, Weigert SP. Violência Obstétrica: A verdadeira dor do parto. Revista Gestão \& Saúde (ISSN 1984-8153).2018. [Citado em 01 de julho de 2020]. Disponível em: http://www.herrero.com.br/files/revista/file2a3ed78d60260c2a5bedb38362615527.pdf .

14. Oliveira MSM. Humanização do parto. Nasce o respeito. Informações práticas sobre seus direitos. Recife: Assessoria Ministerial de Comunicação; Comitê Estadual de Mortalidade Materna de Pernambuco, Procuradoria Geral de Justiça, 2015. [Acesso em: 2020 jul 15] Disponivel em: http://repositorio.asces.edu.br/ handle/123456789/2318.

15. Gabriel AD, Alves VH, Pereira AV, Marchiori GRS, Duarte MR, Rodrigues DP. Percepções de profissionais frente à (in) aplicabilidade das políticas na atenção ao parto e nascimento. SaudColetiv (Barueri) [Internet]. $11^{\circ}$ de maio de 2020 [citado $26^{\circ}$ de março de 2021];9(50):1774 - 178. Disponivel em: http://revistas.mpmcomunicacao.com.br/index.php/saudecoletiva/article/ view/155.

16. Zorzam, B. Direito das mulheres no parto: conversando com profissionais da saúde e do direito, 1. ed. São Paulo: Coletivo Feminista de Sexualidade e Saúde, 2016. [Acesso em: 2020 jul 04] Disponivel em: https://www.mulheres.org.br/wp-content/ uploads/2020/02/direito-mulheres-parto.pdf . 